\title{
An optical configuration for fastidious STEM detector calibration and the effect of the objective lens pre-field
}

\author{
Lewys Jones $^{\mathrm{a}, \mathrm{b}^{*}}$, Aakash Varambhia ${ }^{\mathrm{c}}$, Hidetaka Sawada ${ }^{\mathrm{d}, \mathrm{e}}$, Peter D. Nellist $^{\mathrm{c}}$ \\ a School of Physics, Trinity College Dublin, Dublin 2, Ireland \\ ${ }^{b}$ Advanced Microscopy Laboratory, Centre for Research on Adaptive Nanostructures and Nanodevices (CRANN), Dublin 2, Ireland \\ ${ }^{c}$ Department of Materials, University of Oxford, Oxford, OX13PH, UK \\ d JEOL Ltd, 3-1-2 Musashino, Akishima, 196-8558, Tokyo \\ ${ }^{e}$ Electron Physical Sciences Imaging Center, Diamond Light Source Ltd. Didcot, OX110DE, UK \\ * lewys.jones@tcd.ie
}

\begin{abstract}
In the scanning transmission electron microscope, an accurate knowledge of detector collection angles is paramount in order to quantify signals on an absolute scale. Here we present an optical configuration designed for the accurate measurement of collection angles for both image-detectors and energy-loss spectrometers. By deflecting a parallel electron beam, carefully calibrated using a diffraction pattern from a known material, we can directly observe the projection-distortion in the post-specimen lenses of probe-corrected instruments, the three-fold caustic when an image-corrector is fitted, and any misalignment of imaging detectors or spectrometer apertures. We also discuss for the first time, the effect that higher-order aberrations in the objective-lens pre-field has on such an angle-based detector mapping procedure.
\end{abstract}

\section{Second Abstract -- Lay Description}

The scanning transmission electron microscope is capable of taking pictures of materials down to the atomicscale, but we still don't know all the details of the recording systems that take our pictures. In this work, we explore the details of how these signals reach the detectors that build up our images. We develop a method to help microscopists get an even more accurate and reliable match with computer simulated images.

PACS:

Keywords: ADF-STEM, quantitative STEM, detector angle calibration, detector sensitivity measurement, EELS

\section{Introduction \& Background}

The focussed electron probe of the scanning transmission electron microscope (STEM) allows today's materials scientists to probe the structural, chemical and electronic properties of samples down to the atomic level (Krivanek et al. 2010; Lovejoy et al. 2012; Lin et al. 2015). Much of this progress has been achieved through continual improvements to both the hardware and theoretical tools available; however interfacing these two branches requires a further key ability - instrument calibration.

As early as the 1970's the annular dark-field (ADF) scattering cross-sections of single and double atom clusters were first expressed on an absolute scale (Retsky 1974). Efforts to express this cross-section accurately were advanced significantly in the 1990's with the first demonstration of an ADF sensitivity scan (Singhal, Yang, and Gibson 1997), but it wasn't until a decade later, and alongside the development of aberration correction, that this was reliably demonstrated (LeBeau and Stemmer 2008). This is now the basis of a growing field of so-called 'quantitative ADF' with applications in thickness measurement, composition mapping, and nanoparticle-metrology (LeBeau et al. 2008; LeBeau and Stemmer 2008; Rosenauer et al. 2011; Jones et al. 2014). Similarly, in electron energy-loss spectroscopy (EELS), accurate knowledge of the spectrometer's geometrical collection efficiency (acceptance angle) is essential for accurate quantification (Malis, Cheng, and Egerton 1988).

There are many practical aspects that must be considered when attempting to record ADF data on an absolute scale, and a concise table of the known errors is shown in (Jones 2016). Of greatest importance are the measurements of the detector's inner- and outer-angles and its recording sensitivity. The former dictates the appropriate reference simulation needed, while the latter is used for the normalisation of the recorded images themselves. Equally, in EELS the spectrometer acceptance angle, often known as $\beta$, must be accurately known. Here we present an improved method for measure the ADF detector and EELS aperture angles based on a post-specimen ray-tracing approach. 
The vast majority of published ADF sensitivity scans have been recorded using a confocal optical geometry (LeBeau and Stemmer 2008; Fritz et al. 2011; Findlay and LeBeau 2013; MacArthur, Jones, and Nellist 2014). Here the post-specimen lenses are configured as if for conventional transmission electron microscopy (imaging mode), the probe at the sample plane is reimaged in the detector plane and the normal STEM scan-coils raster the probe across the detector, Figure $2 \mathrm{~b}$ ). However, while the confocal approach is appealing to the experimentalist, as it can be selected via a simple mode change, the STEM imaging experiment is instead done with the post-specimen lenses in the diffraction mode. This introduces a disparity between the calibration and the experiment. In terms of scattering angle propagating through the post-specimen optics, in the confocal scan the electron-probe only experiences up to some few tens of milliradians (mrad) of the post-specimen optics, whereas in an imaging experiment we may wish to collect electrons which have been scattered to hundreds of mrad.

Let us consider this disparity in more detail. If the post-specimen electron flux distribution is recorded on a CCD we see the intensity scattered outside the bright-field disc decays with scattering angle. At shorter cameral-lengths an abrupt truncation to the high-angle scattering can be observed; this is known as the 'flux cut-off' (Martinez et al. 2015) and varies depending on several factors including the accelerating voltage and configuration of microscope used (Jones 2016). In instruments with only round-lenses after the specimen, the origin of this cut-off can be attributed to the spherical aberration of the objective lens or to differential pumping apertures (Katz-Boon et al. 2013). If a post-specimen aberration corrector is present, spherical aberration may be corrected out to some few tens of milliradians, but it is not corrected at several hundred milli-radians. For instruments without a post-specimen aberration corrector, the high-angle scattering may be folded back into lower angles by spherical aberration, leading to a caustic and the enhanced halo at the periphery of the flux-images (Martinez et al. 2015; Jones 2016), but for the highest angles this scattering is simply lost. Previously this has been compensated for in quantitative ADF studies by either assuming some 'effective outer angle' (Findlay and LeBeau 2013), or by observing the cut-off directly (Martinez et al. 2015). For instruments that have non-round lenses after the specimen such as an imaging-corrector or in-column energy filter the flux distribution and cut-off becomes even more complex (Jones 2016; Krause et al. 2016).

Alternatively, the ADF detector scan can be acquired with the post-specimen lenses in diffraction mode, similar to that which was used in the now defunct VG dedicated STEM instruments (Singhal, Yang, and Gibson 1997). This is more reliable as it is directly comparable to the configuration for STEM imaging. This so-called 'swung-beam' approach was redeveloped for modern instruments by two groups at approximately the same time; by Jones and co-workers, who formed a parallel-beam at the sample plane using a JEOL-ARM200CF (Jones 2016); and by Krause et al. for an FEI 80/300 G1 (Krause et al. 2016) who used a small probe-forming aperture to produce a narrow beam albeit with a slightly converging shape. Very recently House et al. demonstrated the parallel-beam setup for a JEOL-2100F (House et al. 2017).

In the swung-beam approach a parallel (or near parallel) beam is rocked in angle using the beam-tilt coils (Krause et al.(Krause et al. 2016)) or STEM scan-coils (Jones (Jones 2016) and House et al. (House et al. 2017)), to trace a small disc/spot through the post-specimen optics and onto the ADF detector. This method represents an improvement on the confocal image-mode approach and allows the post-specimen optics to remain in diffraction mode and unchanged from their STEM imaging settings. However, the small-aperture/tilt-coil approach (Krause et al. 2016) requires accurate balancing of the double-deflection tilt coils to produce pure tilt, and the use of a very small aperture to produce a pseudoparallel beam which is then necessarily not the same current as used in the subsequent imaging experiment.

Instead, here we build on earlier work using a fully parallel-beam and the STEM scan-coils (Jones 2016; House et al. 2017), and demonstrate a rigorously calibrated approach for the recording of tilt-based ADF scans. We use a purely parallel-beam at the sample plane using the same probe-forming aperture, and therefore the same beam current, for detector calibration and for STEM imaging. We verify the tilt-shift purity experimentally during the setup, and diffraction from a known sample to precisely calibrate scattering angles. Finally, we evaluate for the first time, the effect of the objective-lens pre-field aberrations on the resulting swung-maps.

\section{Methods}

The results presented here were all recorded using a probe-corrected JEOL ARM200CF; however, the approach described below is general to other columns and manufacturers. Before beginning the ADF calibration procedure the microscope is first aligned as for normal STEM imaging, selecting a specific primary voltage and spot-size. Special attention should be paid to gun-tilt, spot-alignment, coma adjustment and image-shift to give as straight a beam path as possible through the column. 
In all types of instrument, caustic aberrations cause the beam to be folded back at high angles towards more central parts of the detector. When all STEM imaging detectors are retracted and the transmitted electrons are allowed to fall on a CCD an electron flux pattern can be recorded, Figure 1. In these CCD flux-patterns, where a sufficiently thick specimen is used, we observe an abruptly truncated edge immediately preceded with an enhanced ring of intensity (Jones 2016; Krause et al. 2016).

To verify that the image scan is centred on the optic axis, the operator should insert and align a probe-forming aperture to the 'sweet-spot' of the ronchigram defining a bright-field disc. Next, using some moderate thickness of sample, induce some high angle scattering and record this on the CCD; mark the position of the outer cut-off of this flux and bisect this to give the centre of the so-called 'objective lens cut-off'. Next, adjusting image shift, bring the position of the bright-field disc to the centre. After adjusting image-shift, the probe-forming aperture will need to be removed and coma will need to be evaluated/adjusted; these two adjustments must be iterated until the BF disc is at the centre of the OL cut-off and the coma alignment is acceptable. If this alignment has never been performed before for a particular instrument/voltage/spot-size, the adjustments may take some iterations to converge; however, once corrected this optimisation requires only minor tweaking in future.

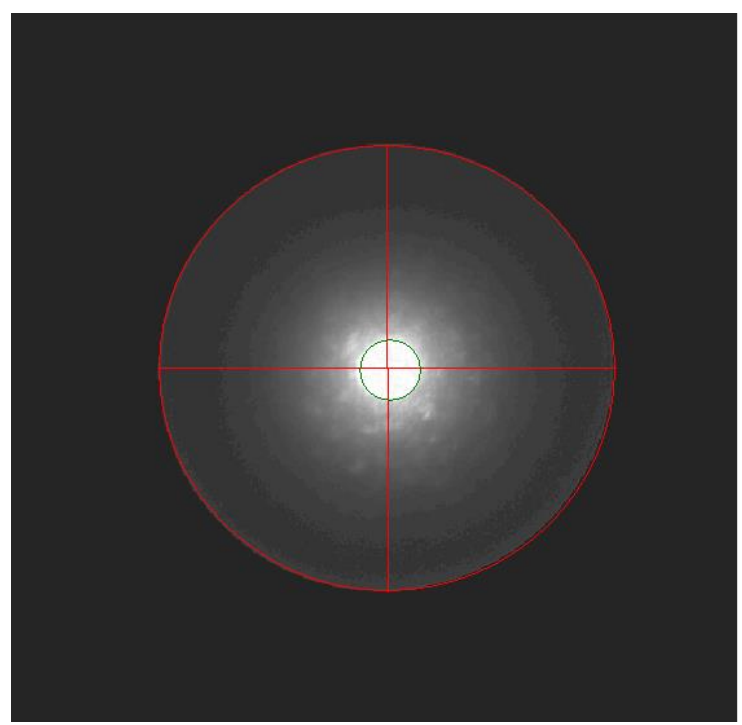

Figure 1. Flux pattern recorded on the CCD. Note the bright-field disc is centred in the objective-lens cut-off.

Once aligned, a probe-forming aperture is inserted to select the flat phase region of the ronchigram and form the STEM probe. So that no additional probe-current scaling is required, the same aperture should be used for the calibration as is used for the associated imaging or spectroscopy. 

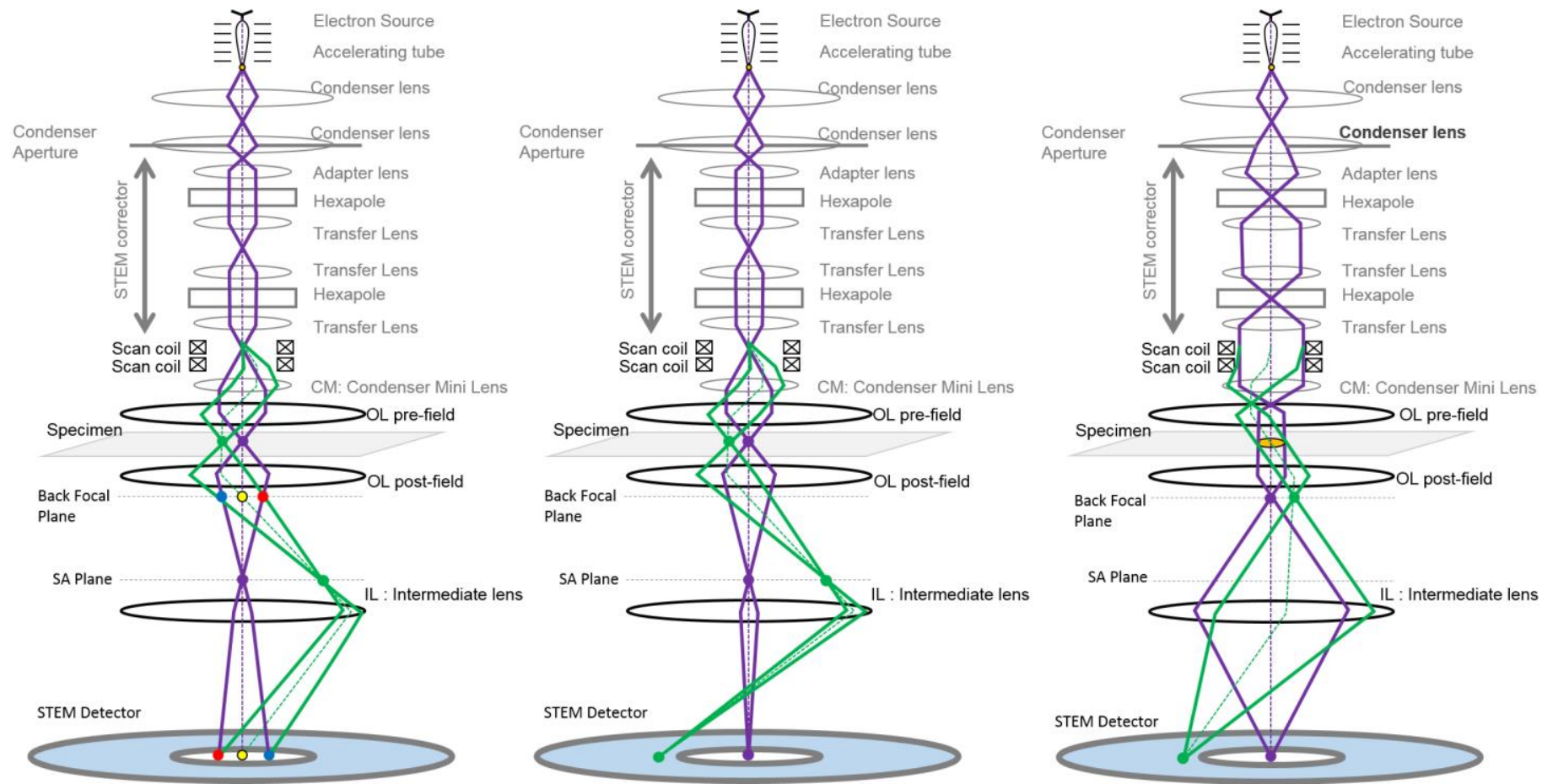

Figure 2. Optical ray diagrams to illustrate a) regular STEM imaging, b) the conventional (confocal) detector scanning approach, and c) the proposed mapping procedure.

Next, a parallel beam with the correct rocking behaviour is created by adjusting the condenser lens system; specifically the final condenser lens (shortened hereafter to 'CL3' for the JEOL-ARM used in this study, though other instruments may use a different shorthand) and the condenser mini-lens (CM), Figure 2. With the post-specimen optics set as for STEM-imaging (JEOL: "Mag mode"; FEI: "Diffraction mode") CL3 is weakened so as to produce a parallel beam at the specimen plane, Figure 3. Beam-current was monitored during this process via the integrated CCD counts, and was found to fluctuate by around $1 \%$ only.
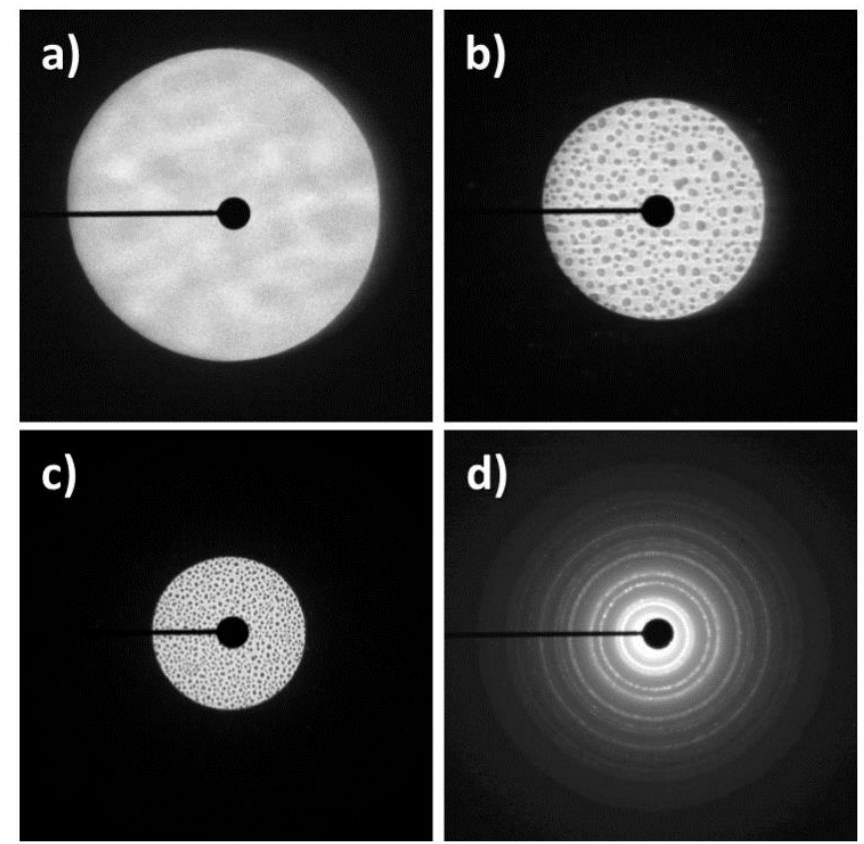

Figure 3. Illustrative examples of the starting, intermediate and final stages in the weakening of CL3. Panel a) shows a typical in focus ronchigram of the carbon film with a probe-forming aperture inserted; as CL3 is weakened, panels b) and c), the field of view in real space increases (more gold balls visible) while the angular space range reduces (probe-forming aperture casts smaller shadow on the CCD).

Next, with the optics set to re-image the STEM-probe on the CCD (JEOL: "Alignment mode"; FEI: "Image mode"), CM is adjusted to observe the minimum disk-wobble during scanning, optimising the cross-over position through the scan coils and ensuring the purest tilt-shift purity behaviour at the sample plane. These two steps are iterated as necessary. 
With a parallel beam now neatly formed, and the post-specimen optics returned to diffraction mode, the key step becomes the calibration of the beam-tilt step afforded by the scan coils. Here we do not rely on any indicated beam-tilt value, rather we perform our own calibration. First, using a known sample, such as the gold aberration-tuning test specimen, a polycrystalline diffraction pattern is recorded, Figure 4 a). This allows us to calibrate the angle-space scaling across the CCD camera by fitting the slope of the diffraction ring radii, Figure $4 \mathrm{c}$ ). Next, with the beam passing through a hole (vacuum) the STEM scan is engaged and a long exposure CCD frame recorded (at least some multiples of the STEM frame time, typically $>40$ s); this is repeated with 90 degrees scan rotation. These two long-exposure frames can be superimposed, Figure $4 \mathrm{~b}$ ), and elegantly reveal the scan-paths traced by the parallel beam as it is rocked in angle about the sample plane; the spacings between the paths equalling precisely one tilt step in $\mathrm{x}$ and $\mathrm{y}$. Necessarily the spacing of these lines depends on the magnification and pixel-sampling of the scan-output of the STEM imaging settings. However, importantly for the practicality of our approach, as this scan makes use of the microscope scan-generator it takes only a few seconds to acquire an entire detector scan rather than several hours (Müller-Caspary et al. 2016). Further, as the same aperture is used for the calibration and STEM imaging, the scan approach is compatible with either the so-called 'dropped gain' or 'dropped-current' approaches described by Jones (Jones 2016), where as always care should be taken to avoid saturation and ensure linearity (LeBeau and Stemmer 2008; Yamashita et al. 2015; Ishikawa et al. 2014).

In practice, it is more convenient for the operator to select the magnification at each camera-length to ensure a sufficient angular field of view for the largest detector being scanned (usually upper dark-field), and then a pixelsampling such that the scan-line patterns on the CCD are clearly resolvable for counting. With these parameters verified the diffraction pattern and scan-path measurements are made for each required camera-length setting. For the scan-line recording, a large post-specimen aperture was inserted to stop high-angle caustic scattering reaching the CCD (hence the circular mask); while for the diffraction pattern recording, no aperture was inserted to allow the maximum possible number of rings to be indexed.
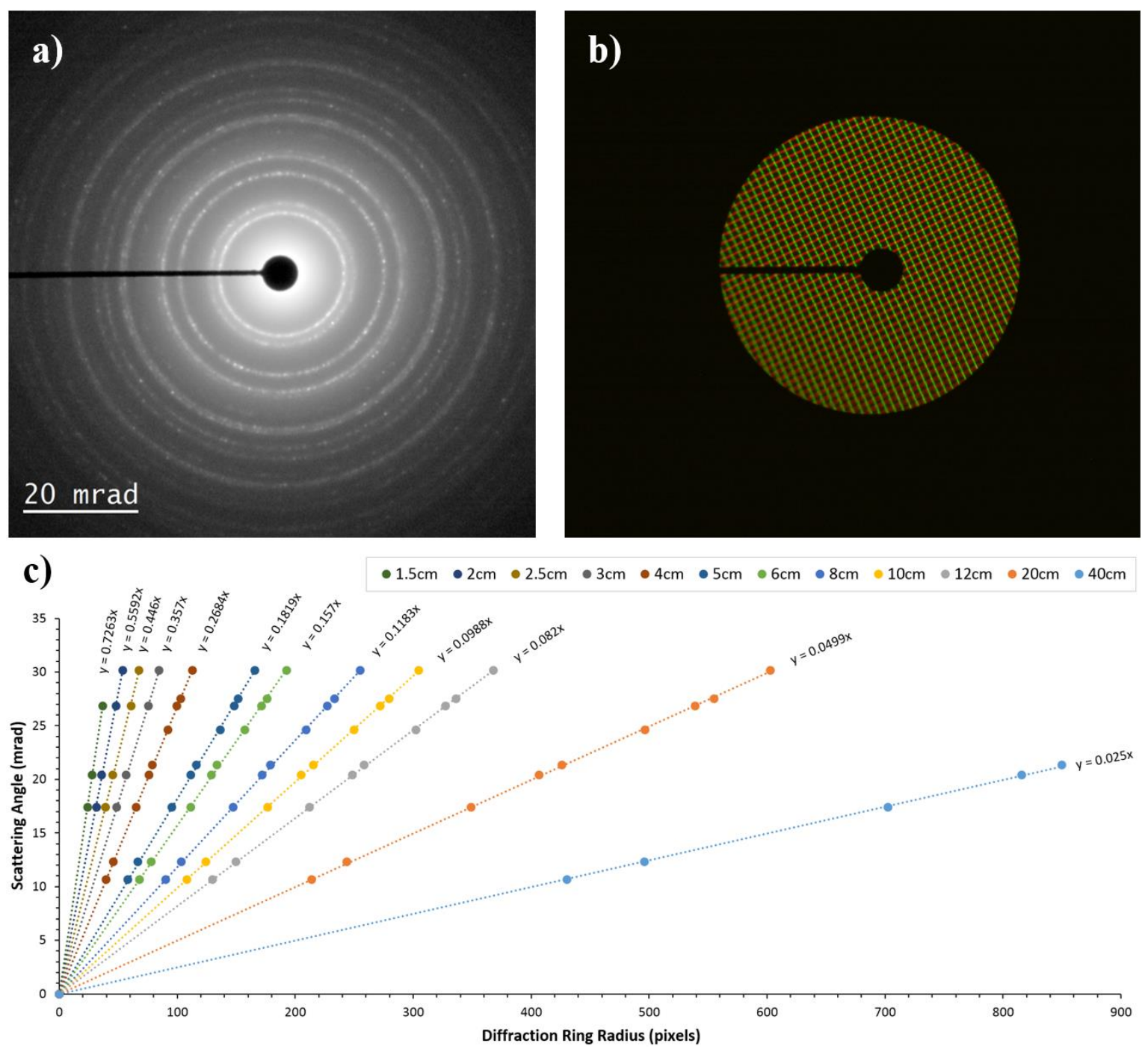
Figure 4. Steps in the angular scan calibrations. For each camera-length, a polycrystalline gold diffraction pattern is recorded (a) [log intensity shown] and indexed (c) to determine the "mrad per CCD-pix". Next, with no sample in the beam path, the swung scans (b) are used to calibrate the "CCD-pix per scan-step". Together these give the "mrad per scan-step". Note the shadow of the beam-stop remains visible in both.

Now that the beam-tilt step has been calibrated, and the appropriate magnification and pixel-sampling selected, a very small adjustment is made to CL3 to increase its strength. This produces a beam which is converging by a small fraction of a milli-radian. The purpose of this is to deliberately blur the observed scan-lines in the long exposure CCD recordings. This ensures that the sensitivity of the ADF detector is scanned in a representative fashion. This operation is shown in Figure 5 and is analogous to a scanning electron microscopist choosing a probe-size that best matches their virtual pixel-size.

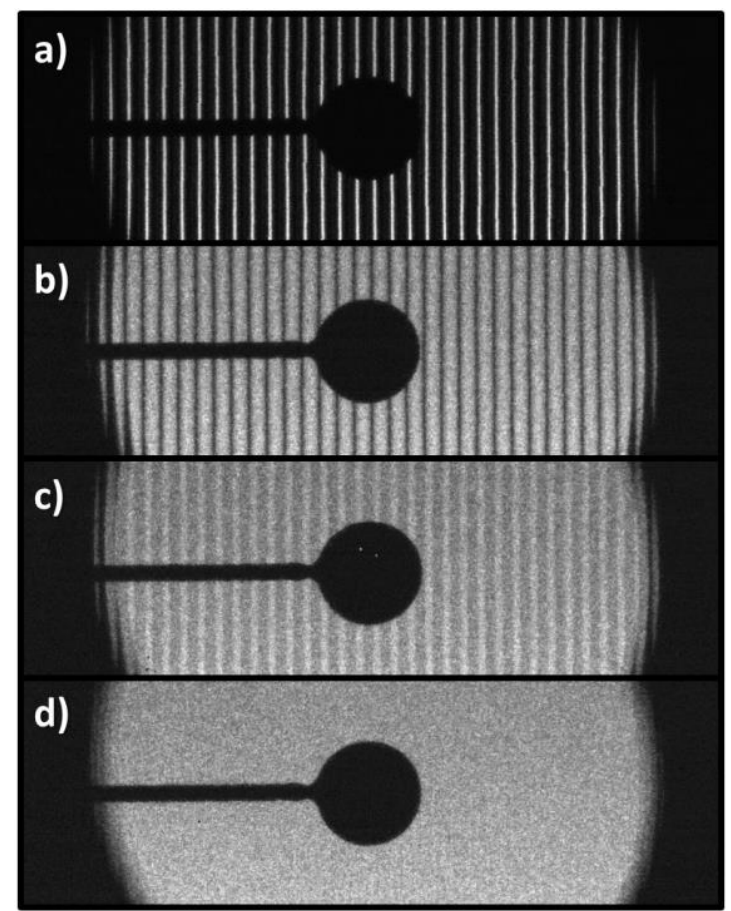

Figure 5. Example of a long exposure CCD frame of the angle-swung beam with a) perfectly parallel beam, and $b)-d)$ with increasing but still minute convergence.

With the beam-tilt step calibrated, the STEM detectors can be scanned and this calibration applied. The recorded STEM-image is then a direct angle-space sensitivity map of the detector. As with conventional confocal detector scanning it should be noted that the operator should make a deliberate, and more importantly a consistent, choice to use either the dropped-current or dropped-gain approaches (Jones 2016).

To calibrate EELS entrance aperture sizes, the same procedure as above was repeated, however rather than taking angle measurements from a detector-signal image, the zero-loss peak intensity is observed in a spectrum image. Not only does this approach allow for the precise acceptance angle to be measured, but also for simultaneous measurement of the homocentricity of the Gatan dark-field detector commonly used during spectrum imaging.

\section{Results}

\section{Direct detector angle measurement}

One of the largest sources of potential error when in ADF quantification lies with mismeasurement of the detector inner-angle (Jones 2016). Previous methods to calibrate the inner angle have included observing the shadow of the hole on the CCD, or slightly better to use a deflected bright-field disc to determine the start of the active layer (LeBeau and Stemmer 2008). However, both these methods may lead to errors; in the former any liner-tube or dead-layer is ignored (or at best another scaling has to be introduced), while in the latter a series of images have to be recorded, superimposed and scrutinised to determine the angle. Instead, the angular scan proposed here directly records the inner angle, Figure 6. This scan directly highlights the presence of any 'dead-layer' on the inside edge of the ADF detector. In Figure 6 the mechanical hole is labelled as the inner-most dashed line. This is what would be observed as a shadow on a CCD, whereas the start of the active layer (outer-most dashed line) is the more representative inner-angle for electron detection. 


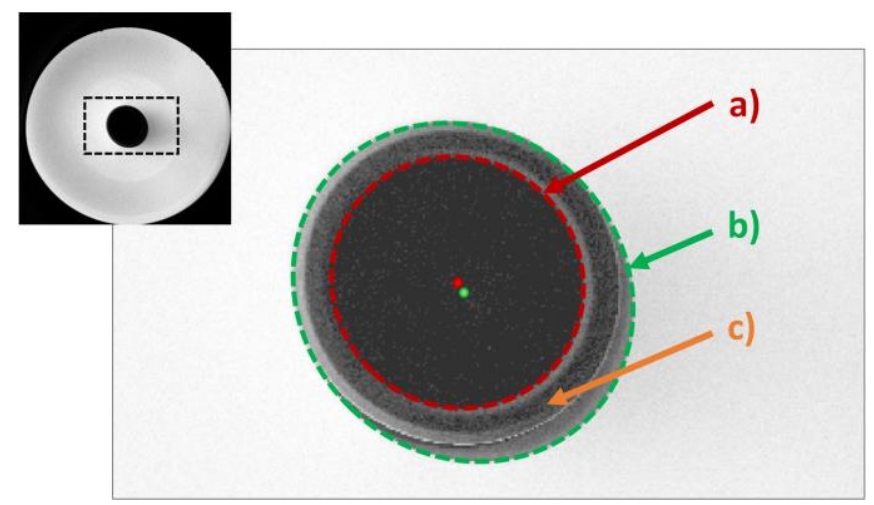

Figure 6. Centre portion of the upper dark-field detector showing a) the inner edge of the drift-tube, $b$ ) the start of the active area of the scintillator, and c) the 'dead layer' between a) and b). The small dots indicate the geometric centres of the two measurement approaches.

While inner-angle mismeasurement is the dominant error in ADF quantification, misinterpretation of the centre of the active region (misalignment of the bright-field disc) can also lead to errors (Jones 2016; Krause et al. 2016).

\section{Direct Hardware Adjustment}

Many STEM instruments make use of multiple imaging detectors simultaneously, however, the quality of this data depends on the accuracy of the mechanical alignment of such hardware. ADF detectors generally have some degree of mechanical adjustability and this is often performed while observing the shadow of their inner angle on a viewing screen or a CCD. However, as described above and in the literature (LeBeau and Stemmer 2008; Martinez et al. 2015), the centre of the detector hole shadow may not represent the active area; furthermore, the Gatan dark-field detector being immediately before the EELS spectrometer acceptance aperture is beyond the viewing screen devices.

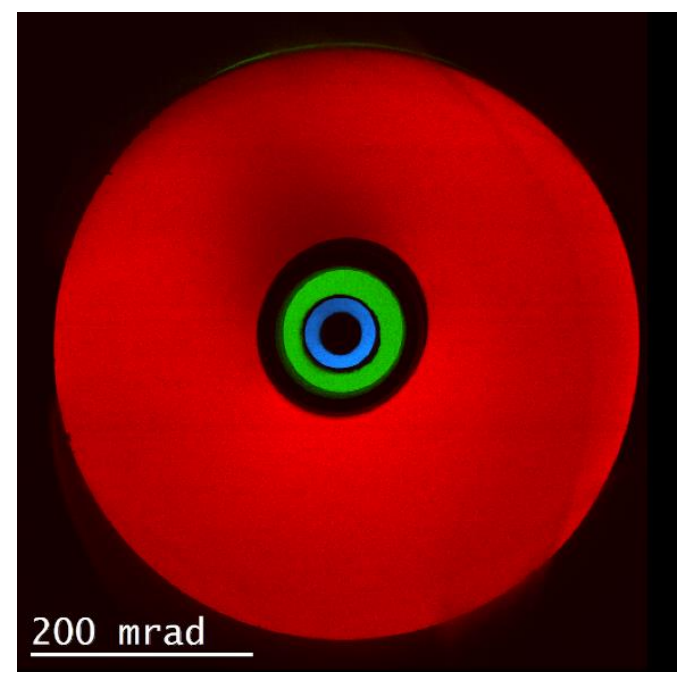

Figure 7. Direct visualisation of the centring of multiple annular detectors; here the upper and lower JEOL ADF detectors (red and green) were mechanically aligned onto the Gatan ADF (blue) which cannot be moved.

Figure 7 shows the superimposed view of the three annular detectors installed in the JEOL ARM200CF used in this study. The only detector which cannot be mechanically adjusted is the one at the entrance to the EELS spectrometer; using the method described in this work all three detectors can be observed simultaneously and the two JEOL annular detectors can be mechanically aligned onto the third. Once established, this configuration allows for the recording of either three simultaneous dark-field signals or two dark-field and one annular bright-field (ABF). Further, in both these cases, it remains possible to record EELS data as no beam stopper is required for forming the ABF image.

\section{Direct EELS entrance aperture measurement}

To directly measure the EELS acceptance apertures, spectrum images were recorded from which a $25 \mathrm{eV}$ region encompassing the zero-loss peak was integrated, Figure 8. 


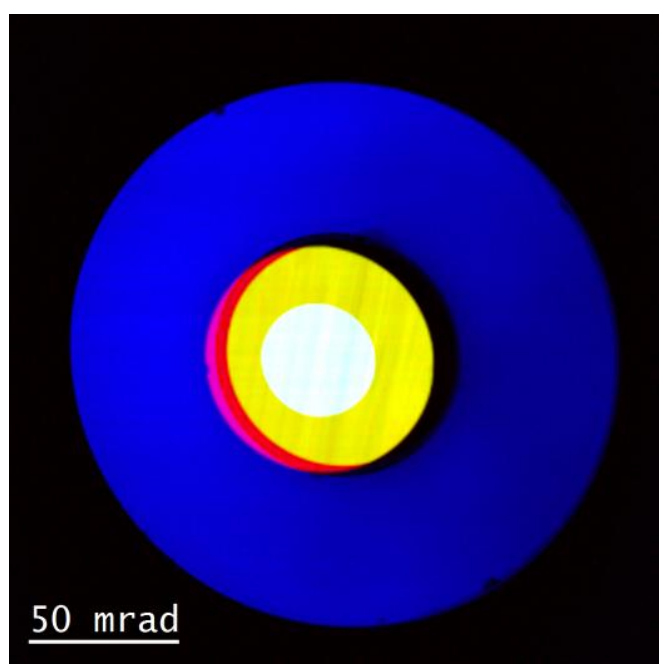

Figure 8. Direct visualisation of the Gatan dark-field (GDF) detector (blue) and various choices of EELS acceptance apertures; the $2.5 \mathrm{~mm}$ aperture (white), the $5 \mathrm{~mm}$ aperture with GDF inserted (yellow), and the extra area of the 5mm aperture revealed with the GDF retracted (red \& magenta).

Spectra were recorded using both the $2.5 \mathrm{~mm}$ and $5 \mathrm{~mm}$ apertures, each with the Gatan DF detector inserted and retracted. In Figure 8, as expected, the $2.5 \mathrm{~mm}$ and $5 \mathrm{~mm}$ apertures are centred with respect to each other. More surprisingly a hardware misalignment was discovered between the Gatan DF detector and these EELS apertures. In the case of the $5 \mathrm{~mm}$ aperture, it was found that part of this is blocked by the GDF (red and magenta area of Figure 8).

The EELS apertures were found to be slightly misaligned from the GatanDF detector meaning some occlusion of the $5 \mathrm{~mm}$ aperture occurs with GDF inserted, Figure 8. This has no mechanical adjustability and so was taken as the centre for the (mechanically adjustable) JEOL detectors above. This centring was optimised (Figure 7) and unlocks valuable multi-detector datasets for the user. For example on the column used here with a $21 \mathrm{mrad}$ objective aperture selected and all three annular detectors inserted, a camera-length of $12 \mathrm{~cm}$ yields simultaneous ABF, LAADF and MAADF data-sets, while at $5 \mathrm{~cm}$ this same configuration yields simultaneous LAADF, MAADF and HAADF.

\section{Observed detector \& Spectrometer Angles}

Using the method described here detector scans were recorded over all the available camera lengths, Figure 9; from these the following inner- and outer-angles were measured, Table 1. 


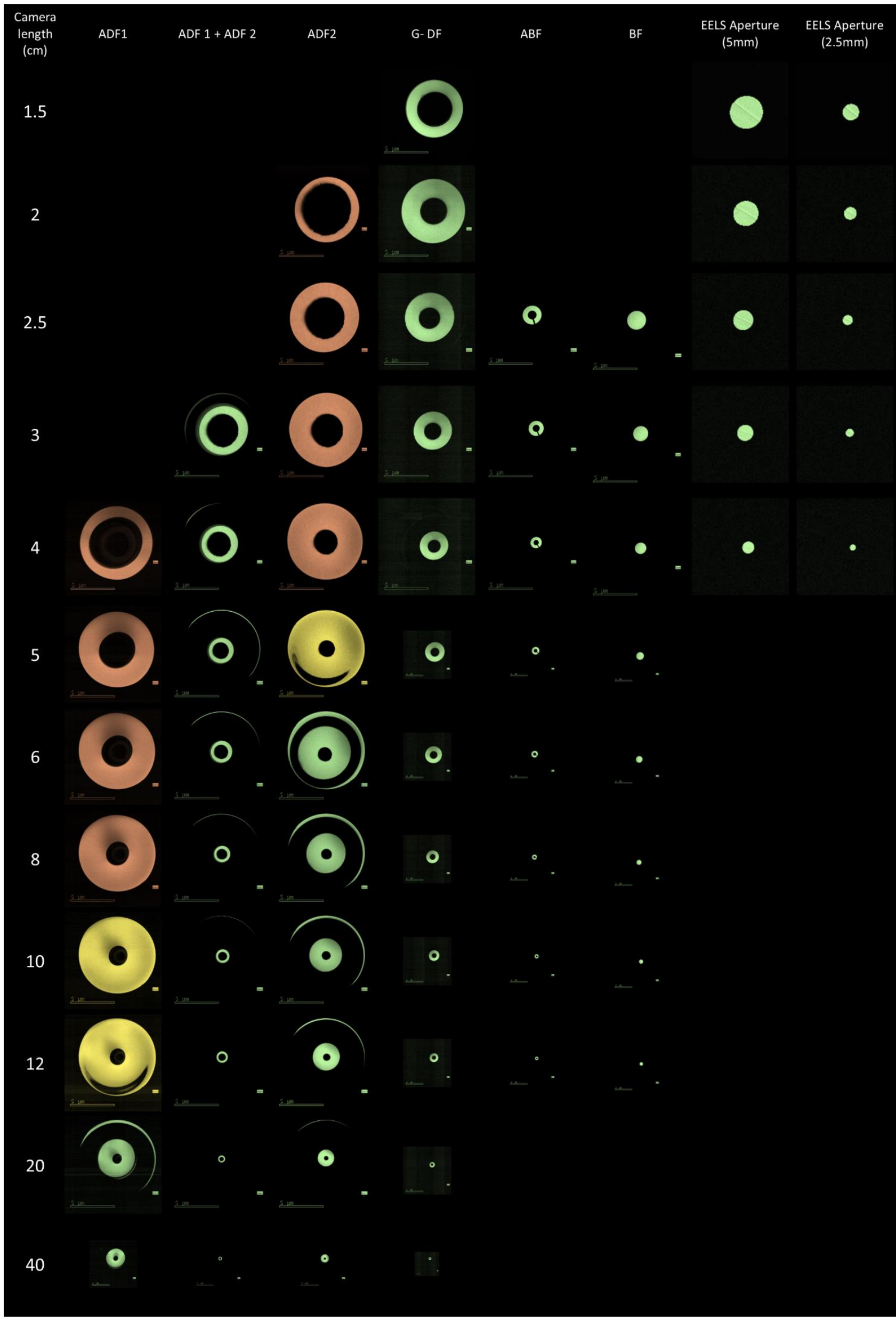

Figure 9. Montage of swung-beam scans across all the available camera-lengths and for various detector configurations shown with the same relative scale; these include the upper dark-field (ADF1), the lower dark-field (ADF2), and the Gatan dark-field (GDF). As a special case, we also show the scans of ADF2 when it is inserted behind ADF1. The JEOL bright field detector (whose outer angle is defined by an aperture) 
Figure 9 shows a montage of all the detector scans recorded from the JEOL ARM200CF studied in this work. We can see that the upper left part of the montage is empty, at these camera-lengths, the respective dark-field detectors occupy such a high angular range that no electrons reach them for a sensitivity scan. Similarly in the very bottom-right of the chart EELS apertures and BF detectors are of little use owing to the incredibly small, and hence inefficient collection. For all the other intermediate camera-lengths the detector scans are shown and assigned a colour based on any need for outer-angle scaling or extrapolation as described in the next section.

\section{The Effect of Higher-order Aberrations in the Objective-lens Pre-field}

Figure 9 shows, from bottom to top, the increase in inner- and outer collection angles that results from reducing the camera-length. If we consider the $\mathrm{ABF}$ detector, this continuously grows in size from bottom to top in this chart. However, if we consider the same trend for the ADF2 detector, this effect is joined by another phenomenon from $12 \mathrm{~cm}$ and shorter where a secondary ring is observed; for ADF1 this ring is observed for $20 \mathrm{~cm}$ and shorter. This effect has also been observed by Krause and co-workers but was attributed to caustic aberrations in the projection system (postspecimen) and was incorporated into their image simulation procedure. While we observe the same 'extra-ring' phenomenon, we demonstrate an alternative explanation - the high-order aberrations of the objective-lens pre-field.

To establish the origin unambiguously, two data must be recorded; firstly we record another swung parallel-beam scan with a very slow pixel dwell-time (for example, $>100 \mathrm{~ms}$ ) and a small number of pixels (32x32 grid) on a longexposure CCD frame, Figure 10 a). With a long dwell-time, the scan-lines shown in Figure 4 b) become discrete points; this allows them to be counted and individual coordinates extracted. Towards the edge of this pattern of dots, we see the points are folded back towards the centre. This folding-back effect can also observed if the same scan as Figure $4 \mathrm{~b}$ ) is performed without a selected-area aperture (see supplementary video 1).
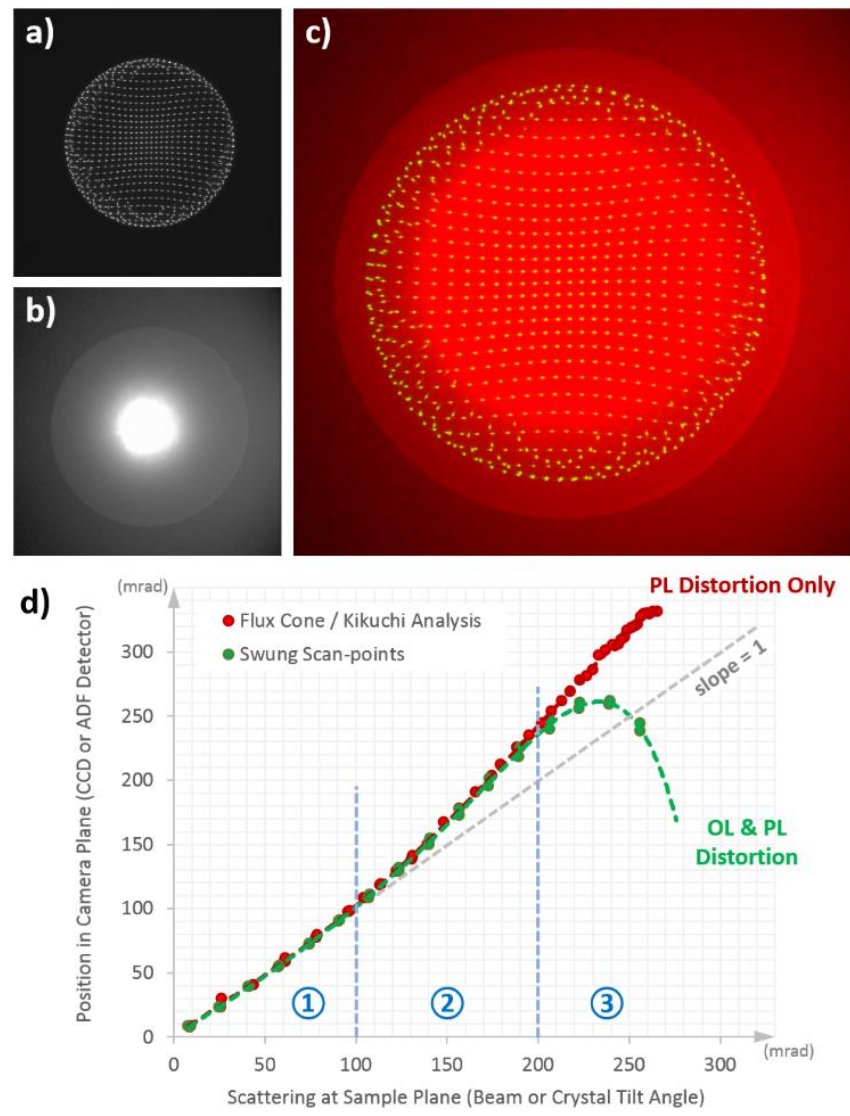

Figure 10. (a) swung parallel-beam point recording as captured on a long-exposure CCD image, and (b) a convergent beam fluxdistribution image on the same CCD. Post specimen lenses were not adjusted between capturing $(a)$ and $(b)$. Panel (c) shows the overlay of $(a)$ and $(b)$ where the difference in the outer-angle reached becomes apparent. Plot (d) shows the point-recording (green circles) versus scatteredflux recording (red circles) at fixed camera-length. The grey dashed line represents a line of unit gradient.

Next, without touching the post-specimen lens settings, we record a flux distribution pattern from some thick region of sample, Figure $10 \mathrm{~b}$ ) (Martinez et al. 2015). Overlaying these two data, Figure $10 \mathrm{c}$ ), shows that the swung-scan 
explores a more limited angular range than the high-angle scattering from an un-tilted beam. This difference was evaluated for a number of camera-lengths and the flux-map was found to extend $19.3 \%$ beyond the swung-mapping in all cases.

Quantifying the scan-point image was done by measuring the position in the camera-plane (on the CCD) and using the angular-calibration of the CCD-pixels from the diffraction ring-patterns (Figure $10 \mathrm{~d}$ ), dashed green line). As the flux-map cannot be indexed in the same way as the point-grid, a different approach must be followed to quantify this data. One possibility is to perform an analysis similar to Craven et al. by fitting the distortion of a Kikuchi pattern (Craven et al. 2017); however, as we require only a one-dimensional profile (round lens only post-specimen column), a physical tilt-series of Kikuchi-map pole figures from a strontium titanate wedge were recorded instead (see supplementary video 2). Here the illumination remains untilted, but rather the sample is physically tilted in a well calibrated goniometer and the position of the pole is used as a reliable measure across angle space. The probe-forming aperture was displaced to the side of the pole, so that only dark-field electrons formed each pole-figure. The position of the Kikuchi-pole was traced with respect to physical sample-tilt of, and is also plotted in Figure $10 \mathrm{~d}$ ). Importantly, this pole-figure was formed using an untilted-beam and its position is dependent on the crystal-tilt and post-specimen aberrations only - the swung point-grid by contrast includes both pre- and post-specimen aberrations.

Several noteworthy observations arise from this figure; firstly, in the region up to $100 \mathrm{mrad}$, both data lie on the line with gradient equal to one. This linearity, and the linearity shown in Figure 4, indicates that projection-distortion is minimal in this range. Second, in the region 100-200 mrad, both plots deviate upward as a result of projection distortion (also observed by Craven et al. (Craven et al. 2017)) but remain coincident with each other. Thirdly, and perhaps most importantly, the two curves deviate from one another for sample-plane tilts greater than $200 \mathrm{mrad}$. While the beam-tilt point-map is affected by both OL and PL high-order aberrations, the Kikuchi-tilt-series is only affected by post-specimen aberrations and continues to increase monotonically up to around $270 \mathrm{mrad}$ before becoming truncated by some very high-order aberration in the projection system (Martinez et al. 2015). The swung-point-grid analysis on the other hand being affected by both pre- and post-specimen aberrations is seen to fold back on itself; this folding is the true cause of the 'extra-ring' observed by Krause et al and House et al. (Krause et al. 2016; House et al. 2017).

\section{ADF scans through TEM-correctors}

The beam paths for high-angle electrons become even more complicated in instruments fitted with post-specimen (image) aberration correctors. These correctors are not designed to accept scattering out into the hundreds of milliradians. In instruments with image-correctors this abrupt truncation becomes more triangular as the circular objectivelens truncation is distorted thought the six-fold optics (three-fold parasitic) of the imaging-corrector; again, this effect has been observed equally in instruments from JEOL (Jones et al. 2014) and FEI (Krause et al. 2016). This same effect persists in angle-swung detector scans where image-correctors were again found to introduce significant distortion in the ADF scans; for this reason, such instruments are not recommended for quantitative ADF experiments.

Figure 11 shows equivalent scans of the same model of JEOL ADF detector both recorded at a $10 \mathrm{~cm}$ camera-length from a probe-corrected and image-corrected instruments at the University of Oxford and JEOL-Akishima respectively.
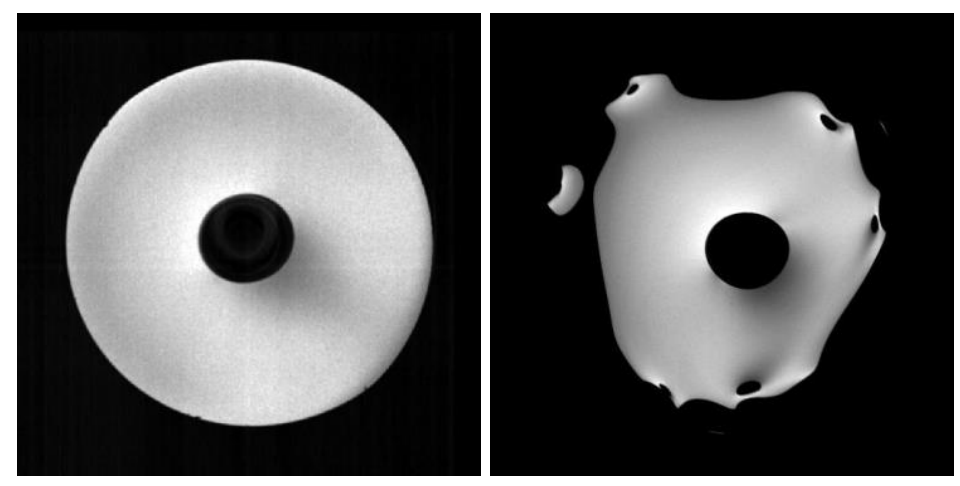

Figure 11. Comparison of swung-beam ADF scans for probe-corrected (left) and TEM-corrected (right) instruments. Both scans were recorded at $10 \mathrm{~cm}$ cameral-length.

While the inner-angle of the detectors is similar, the image-corrected case exhibits a significantly more complicated shape. This is in part due to the parasitic aberration expected from a six-fold corrector design. This severe distortion yields an azimuthally varying outer-angle; however with the flexibility afforded by the projection lens system it is expected that a more optimal configuration can be found in future. For systems with in-column omega type energy filters, this flux distortion becomes even more distorted (Jones 2016). 


\section{Discussion}

The necessity for mapping the STEM imaging detectors in angle-space lies in two main issues; firstly, from the spherical aberration of the post-specimen optics, and secondly in the difficulty of accurately measuring the inner angle of the active region of the detector. With the swung-mapping approach we observe the true ray-tracing behaviour unlike the simple physical geometry observed in confocal mapping.

This result in Figure 10 has several significant implications for STEM detector mapping; each of these, though while not prohibitive, the user should be aware of.

- For measured angles up to around $200 \mathrm{mrad}$, OL aberrations have a negligible effect, and the directly observed swung-map result can be used as is.

- When recording a swung-beam detector scan, phantom extra rings may be observed due to the fold-over effect shown in Figure 10. These only arise as a result of the mapping procedure itself and do not exist in a conventional STEM imaging experiment. These phantom rings should be ignored in the determination of the ADF outer-angle and only the innermost detector image considered.

- Outer angles greater than around 200-210 mrad cannot be mapped using the swung-beam approach alone; however, Kikuchi-tilt analysis shows scattering up to around $270 \mathrm{mrad}$ can reach the camera-plane.

- These outer-angles (>200-210 mrad) should be determined by suitable scaling of valid ones obtained at longer camera-lengths.

- Beyond around $270 \mathrm{mrad}$ (for this system) the very high order aberrations in the projection system truncate the flux reaching the ADF and this can be considered an upper limit to the scattering necessary to consider in image simulation.

\section{The Effective Detector Angles}

Taking into account the above effect of higher-order aberrations, the effective outer angles may differ from those directly observed during the mapping. The resultant chart for the experimentalist is shown below.

\begin{tabular}{|c|c|c|c|c|c|c|c|c|c|c|c|c|}
\hline \multirow[b]{3}{*}{ CL } & \multicolumn{2}{|c|}{ Upper ADF } & \multicolumn{3}{|c|}{ Lower ADF } & \multicolumn{3}{|c|}{ Gatan DF } & \multicolumn{2}{|c|}{$\mathbf{B F} / \mathbf{A B F}$} & \multicolumn{2}{|c|}{$\begin{array}{c}\text { EELS Aperture } \\
\text { (outer angle) }\end{array}$} \\
\hline & \multirow[b]{2}{*}{ Inner } & \multirow[b]{2}{*}{ Outer } & \multirow[b]{2}{*}{ Inner $^{\S}$} & \multicolumn{2}{|c|}{ Outer } & \multirow[b]{2}{*}{ Inner } & \multicolumn{2}{|c|}{ Outer } & \multirow[b]{2}{*}{ Inner } & \multirow[b]{2}{*}{ Outer } & \multirow[b]{2}{*}{$5 \mathrm{~mm}$} & \multirow[b]{2}{*}{$2.5 \mathrm{~mm}$} \\
\hline & & & & $\begin{array}{l}\text { (behind } \\
\text { ADF1) }\end{array}$ & (solo) & & $\begin{array}{l}\text { (behind } \\
\text { ADF2) }\end{array}$ & (solo) & & & & \\
\hline $\begin{array}{l}1.5 \\
\mathrm{~cm}\end{array}$ & & & & & & $\begin{array}{l}109.07 \\
\pm 0.58 \\
\end{array}$ & $\begin{array}{r}176.30 \\
\pm 1.54 * \\
\end{array}$ & $\begin{array}{r}176.30 \\
\pm 1.54 * \\
\end{array}$ & & & $\begin{array}{l}101.69 \\
\pm 0.14 \\
\end{array}$ & $\begin{array}{r}51.15 \\
\pm 0.14 \\
\end{array}$ \\
\hline $\begin{array}{c}2 \\
\mathrm{~cm}\end{array}$ & & & $\begin{array}{l}155.24 \\
\pm 3.80\end{array}$ & & $271 *$ & $\begin{array}{r}82.30 \\
\pm 0.21\end{array}$ & $\begin{array}{l}135.93 \\
\pm 0.39\end{array}$ & $\begin{array}{l}194.15 \\
\pm 2.21\end{array}$ & & & $\begin{array}{l}77.37 \\
\pm 0.43\end{array}$ & $\begin{array}{l}38.12 \\
\pm 0.89\end{array}$ \\
\hline $\begin{array}{l}2.5 \\
\mathrm{~cm}\end{array}$ & & & $\begin{array}{l}127.13 \\
\pm 1.94 \\
\end{array}$ & & $271 *$ & $\begin{array}{r}67.63 \\
\pm 0.59 \\
\end{array}$ & $\begin{array}{l}111.31 \\
\pm 1.09 \\
\end{array}$ & $\begin{array}{l}153.60 \\
\pm 4.64 \\
\end{array}$ & $\begin{array}{r}27.06 \\
\pm 0.07 \\
\end{array}$ & $\begin{array}{r}58.79 \\
\pm 0.37 \\
\end{array}$ & $\begin{array}{r}62.38 \\
\pm 0.29 \\
\end{array}$ & $\begin{array}{r}32.14 \\
\pm 0.11 \\
\end{array}$ \\
\hline \begin{tabular}{c|}
3 \\
$\mathrm{~cm}$
\end{tabular} & & & $\begin{array}{l}100.91 \\
\pm 1.06 \\
\end{array}$ & $\begin{array}{l}150.55 \\
\pm 5.76 \\
\end{array}$ & $271 *$ & $\begin{array}{l}53.06 \\
\pm 0.53 \\
\end{array}$ & $\begin{array}{l}88.51 \\
\pm 0.98 \\
\end{array}$ & $\begin{array}{l}117.90 \\
\pm 2.66 \\
\end{array}$ & $\begin{array}{l}21.96 \\
\pm 0.18 \\
\end{array}$ & $\begin{array}{l}46.53 \\
\pm 0.28 \\
\end{array}$ & $\begin{array}{l}49.19 \\
\pm 0.43 \\
\end{array}$ & $\begin{array}{r}24.94 \\
\pm 0.18 \\
\end{array}$ \\
\hline $\begin{array}{c}4 \\
\mathrm{~cm}\end{array}$ & $\begin{array}{l}166.55 \\
\pm 1.03 \\
\end{array}$ & $271 *$ & $\begin{array}{r}77.43 \\
\pm 0.88 \\
\end{array}$ & $\begin{array}{l}117.77 \\
\pm 4.46 \\
\end{array}$ & $271 *$ & $\begin{array}{l}40.96 \\
\pm 0.15\end{array}$ & $\begin{array}{r}67.39 \\
\pm 0.28 \\
\end{array}$ & $\begin{array}{l}90.52 \\
\pm 2.69 \\
\end{array}$ & $\begin{array}{r}16.96 \\
\pm 0.01 \\
\end{array}$ & $\begin{array}{l}35.70 \\
\pm 0.21 \\
\end{array}$ & $\begin{array}{c}37.94 \\
\pm 0.33 \\
\end{array}$ & $\begin{array}{r}19.28 \\
\pm 0.02\end{array}$ \\
\hline \begin{tabular}{c|}
5 \\
$\mathrm{~cm}$
\end{tabular} & $\begin{array}{l}111.80 \\
\pm 0.22\end{array}$ & $271 *$ & $\begin{array}{l}52.37 \\
\pm 0.29\end{array}$ & $\begin{array}{c}79.31 \\
\pm 2.04 \dagger\end{array}$ & $198.01 * \dagger$ & $\begin{array}{l}27.64 \\
\pm 0.38\end{array}$ & $\begin{array}{l}46.17 \\
\pm 0.70\end{array}$ & $\begin{array}{l}61.48 \\
\pm 1.68\end{array}$ & $\begin{array}{r}10.99 \\
\pm 0.05\end{array}$ & $\begin{array}{l}24.41 \\
\pm 0.23\end{array}$ & & \\
\hline \begin{tabular}{c|}
6 \\
$\mathrm{~cm}$
\end{tabular} & $\begin{array}{l}97.14 \\
\pm 0.87\end{array}$ & $271 *$ & $\begin{array}{l}44.40 \\
\pm 0.02\end{array}$ & $\begin{array}{c}69.58 \\
\pm 0.89 \dagger\end{array}$ & $\begin{array}{c}164.47 \\
\pm 7.32 \dagger\end{array}$ & $\begin{array}{l}24.04 \\
\pm 0.04\end{array}$ & $\begin{array}{l}39.02 \\
\pm 0.07\end{array}$ & $\begin{array}{l}52.75 \\
\pm 0.81\end{array}$ & $\begin{array}{c}9.45 \\
\pm 0.03\end{array}$ & $\begin{array}{l}20.65 \\
\pm 0.30\end{array}$ & & \\
\hline \begin{tabular}{c|}
8 \\
$\mathrm{~cm}$
\end{tabular} & $\begin{array}{r}72.80 \\
\pm 1.08 \\
\end{array}$ & $271 *$ & $\begin{array}{c}33.01 \\
\pm 0.36\end{array}$ & $\begin{array}{c}51.37 \\
\pm 0.91 \dagger\end{array}$ & $\begin{array}{c}120.77 \\
\pm 4.32 \dagger\end{array}$ & $\begin{array}{c}17.48 \\
\pm 0.36\end{array}$ & $\begin{array}{l}29.35 \\
\pm 0.66 \\
\end{array}$ & $\begin{array}{l}39.02 \\
\pm 0.47 \\
\end{array}$ & $\begin{array}{c}6.71 \\
\pm 0.01 \\
\end{array}$ & $\begin{array}{c}15.62 \\
\pm 0.20 \\
\end{array}$ & & \\
\hline $\begin{array}{c}10 \\
\mathrm{~cm}\end{array}$ & $\begin{array}{c}61.72 \\
\pm 1.61 \\
\end{array}$ & $244.84 *$ & $\begin{array}{c}28.13 \\
\pm 0.07 \\
\end{array}$ & $\begin{array}{c}44.24 \\
\pm 0.72 \\
\end{array}$ & $\begin{array}{c}104.36 \\
\pm 3.76 \dagger \\
\end{array}$ & $\begin{array}{r}15.35 \\
\pm 0.05 \\
\end{array}$ & $\begin{array}{r}24.82 \\
\pm 0.09 \\
\end{array}$ & $\begin{array}{l}33.53 \\
\pm 0.64 \\
\end{array}$ & $\begin{array}{c}6.03 \\
\pm 0.09 \\
\end{array}$ & $\begin{array}{r}13.20 \\
\pm 0.28 \\
\end{array}$ & & \\
\hline $\begin{array}{c}12 \\
\mathrm{~cm}\end{array}$ & $\begin{array}{r}50.39 \\
\pm 1.29 \\
\end{array}$ & $199.89 * \dagger$ & $\begin{array}{r}22.85 \\
\pm 0.02 \\
\end{array}$ & $\begin{array}{c}36.00 \\
\pm 0.36 \\
\end{array}$ & $\begin{array}{c}84.72 \\
\pm 3.22 \dagger \\
\end{array}$ & $\begin{array}{r}12.25 \\
\pm 0.12 \\
\end{array}$ & $\begin{array}{l}19.94 \\
\pm 0.22 \\
\end{array}$ & $\begin{array}{l}27.38 \\
\pm 0.52 \\
\end{array}$ & $\begin{array}{c}4.82 \\
\pm 0.01 \\
\end{array}$ & $\begin{array}{r}10.98 \\
\pm 0.12 \\
\end{array}$ & & \\
\hline \begin{tabular}{c|}
20 \\
$\mathrm{~cm}$
\end{tabular} & $\begin{array}{c}30.33 \\
\pm 0.76\end{array}$ & $\begin{array}{c}114.38 \\
\pm 4.33 \dagger\end{array}$ & $\begin{array}{c}13.57 \\
\pm 0.08\end{array}$ & $\begin{array}{l}22.02 \\
\pm 0.25\end{array}$ & $\begin{array}{c}50.95 \\
\pm 1.44 \dagger\end{array}$ & $\begin{array}{c}7.32 \\
\pm 0.13\end{array}$ & $\begin{array}{r}11.79 \\
\pm 0.24\end{array}$ & $\begin{array}{l}16.29 \\
\pm 0.36\end{array}$ & & & & \\
\hline \begin{tabular}{l|}
40 \\
$\mathrm{~cm}$
\end{tabular} & $\begin{array}{r}14.75 \\
\pm 0.13\end{array}$ & $\begin{array}{l}58.74 \\
\pm 2.30\end{array}$ & $\begin{array}{c}6.64 \\
\pm 0.10\end{array}$ & $\begin{array}{c}11.04 \\
\pm 0.16\end{array}$ & $\begin{array}{l}25.78 \\
\pm 0.92\end{array}$ & $\begin{array}{c}3.07 \\
\pm 0.01\end{array}$ & $\begin{array}{c}6.01 \\
\pm 0.02\end{array}$ & $\begin{array}{c}8.60 \\
\pm 0.07\end{array}$ & & & & \\
\hline
\end{tabular}


Table 1. Detector angles measured using the swung diffraction method for the Oxford JEOL ARM200CF. For the lower ADF and the Gatan DF, outer angles are given both for detectors inserted alone, and with shadowing from the detectors above. The colour scheme represents the result of directly measuring angles below 100 mrad in green, correcting for PL mag-distortion in yellow, and extrapolating from other camera-lengths in orange.

To evaluate more quantitatively the effect of not incorporating the projection-distortion on ADF sensitivity, comparison simulations can be performed. Using a classical confocal analysis for the JEOL-ARM instrument evaluated above, combined with a flux-weighting from a CCD flux-map (Martinez et al. 2015), and operated at a $12 \mathrm{~cm}$ cameralength we would measure a detector angle of 53.2-132.2 mrad, whereas with the full angle-mapped method this same camera-length yields detector angles of 50.39-199.89 mrad. While the agreement in the inner angles is reasonable (far more limited distortions are present at low angles), the disagreement in the outer angle is significantly worse. This would lead to an incorrect overestimate in the evaluation of a sample-thickness, in for example a platinum wedge, by up to $30 \%$. Alternatively a confocal detector analysis without flux-weighting (outer angle simply scaled from the inner angle (MacArthur, Jones, and Nellist 2014)) would yield an underestimate of around 15-30\% similar to other studies (Findlay and LeBeau 2013; Krause et al. 2016).

Fortunately, armed with the above table, and with the range of detectors and camera-lengths available the operator has a good degree of flexibility; for example, if the operator is dissatisfied with the inner-edge homogeneity (Findlay and LeBeau 2013) of ADF1 at 8cm (72.80-271 mrad) they can instead select ADF2 at 4cm (77.43-271 mrad). If the user wants an alternative to the BF-plus-beam-stop approach to recording ABF data at $6 \mathrm{~cm}(9.45-20.65 \mathrm{mrad})$, they can select the GDF at $12 \mathrm{~cm}(12.25-19.94 \mathrm{mrad})$. We also see that using a $2.5 \mathrm{~mm}$ EELS aperture at a $2 \mathrm{~cm}$ camera-length $(38.12 \mathrm{mrad})$ is similar to using the $5 \mathrm{~mm}$ at $4 \mathrm{~cm}$ (37.94 mrad) except at $4 \mathrm{~cm}$ the ADF2 imaging signal becomes far more efficient. Significant variations in ADF sensitivity exist between detectors and instruments meaning that measurements must be made specifically for each configuration used (MacArthur, Jones, and Nellist 2014). The values here may be similar to other installations of the same model but operators should repeat the method described here for their own column.

\section{Conclusions}

Here we have presented a method for the direct angular-space observation of the sensitivity of imaging detectors and EELS acceptance apertures in the STEM. We describe a method using a parallel beam swung in angle space with careful calibrations of both the angular step and tilt-shift purity or our measurements. The same condenser aperture is used for both imaging experiments and the calibrations ensuring direct correspondence or beam currents for quantitative ADF imaging work.

Extending the technique to integrated-ZLP scans directly reveals the inner angles of EELS acceptance apertures including any shadowing from the nearby Gatan dark-field detector.

For probe-corrected instruments the effects of projector-lens distortion were observed for some camera-lengths, and a scaling was evaluated to allow the appropriate reference simulations to still be performed. Additionally, the number of detectors and camera-lengths available now mean that alternatives can be selected to avoid the problematic sensitivity responses. For image-corrected instruments, serious artefacts can be observed in the angular detector sensitivity response which greatly complicate the preparation of STEM reference simulations.

\section{Acknowledgments}

The research leading to these results has received funding from the European Union Seventh Framework Programme under Grant Agreement 312483 - ESTEEM2 (Integrated Infrastructure Initiative-I3) and EPSRC grant code, EP/K040375/1, for the 'South of England Analytical Electron Microscope'. LJ \& AV also acknowledge funding from JEOL UK and Johnson Matthey. The authors would like to acknowledge Gerardo Martinez for useful discussions when preparing the manuscript.

\section{References}

Craven, Alan J., Hidetaka Sawada, Sam McFadzean, and Ian MacLaren. 2017. "Getting the Most out of a Post-Column EELS Spectrometer on a TEM/STEM by Optimising the Optical Coupling." Ultramicroscopy 180 (September). Elsevier B.V.:66-80. https://doi.org/10.1016/j.ultramic.2017.03.017.

Findlay, S.D., and J.M. LeBeau. 2013. "Detector Non-Uniformity in Scanning Transmission Electron Microscopy." 
Ultramicroscopy 124 (January):52-60. http://dx.doi.org/10.1016/j.ultramic.2012.09.001.

Fritz, R, A Beyer, W Stolz, K Müller, M Schowalter, A. Rosenauer, I Häusler, et al. 2011. "Quantitative Analysis of Chemical Composition Using HAADF-STEM in a JEOL 2200FS.” Microscopy and Microanalysis 17 (S2):141011. https://doi.org/10.1017/S1431927611007926.

House, Stephen D., Yuxiang Chen, Rongchao Jin, and Judith C. Yang. 2017. "High-Throughput, Semi-Automated Quantitative STEM Mass Measurement of Supported Metal Nanoparticles Using a Conventional TEM/STEM." Ultramicroscopy 182:145-55. https://doi.org/10.1016/j.ultramic.2017.07.004.

Ishikawa, Ryo, A.R. Lupini, S.D. Findlay, and Stephen J Pennycook. 2014. "Quantitative Annular Dark Field Electron Microscopy Using Single Electron Signals.” Microscopy and Microanalysis : The Official Journal of Microscopy Society of America, Microbeam Analysis Society, Microscopical Society of Canada 20 (1). Cambridge University Press:99-110. https://doi.org/10.1017/S1431927613013664.

Jones, L. 2016. "Quantitative ADF STEM: Acquisition, Analysis and Interpretation." IOP Conference Series: Materials Science and Engineering 109 (1). IOP Publishing:12008. https://doi.org/10.1088/1757-899X/109/1/012008.

Jones, L., K.E. MacArthur, V.T. Fauske, Antonius T J van Helvoort, and Peter D Nellist. 2014. "Rapid Estimation of Catalyst Nanoparticle Morphology and Atomic-Coordination by High-Resolution Z-Contrast Electron Microscopy." Nano Letters 14 (11). American Chemical Society:6336-41. https://doi.org/10.1021/n1502762m.

Katz-Boon, Hadas, Chris J Rossouw, C. Dwyer, and J. Etheridge. 2013. "Rapid Measurement of Nanoparticle Thickness Profiles.” Ultramicroscopy 124 (January). Elsevier:61-70. https://doi.org/10.1016/j.ultramic.2012.08.009.

Krause, Florian F., Marco Schowalter, Tim Grieb, Knut Müller-Caspary, Thorsten Mehrtens, and A. Rosenauer. 2016. "Effects of Instrument Imperfections on Quantitative Scanning Transmission Electron Microscopy." Ultramicroscopy 161. Elsevier:146-60. https://doi.org/10.1016/j.ultramic.2015.10.026.

Krivanek, O L, M F Chisholm, V. Nicolosi, T.J. Pennycook, George J Corbin, N Dellby, M Murfitt, et al. 2010. “Atomby-Atom Structural and Chemical Analysis by Annular Dark-Field Electron Microscopy." Nature 464 (7288). Macmillan Publishers Limited. All rights reserved:571-74. https://doi.org/10.1038/nature08879.

LeBeau, J.M., S.D. Findlay, L. J. Allen, and S. Stemmer. 2008. "Quantitative Atomic Resolution Scanning Transmission Electron Microscopy." Physical Review Letters 100 (20). American Physical Society:206101-. http://link.aps.org/doi/10.1103/PhysRevLett.100.206101.

LeBeau, J.M., and S. Stemmer. 2008. "Experimental Quantification of Annular Dark-Field Images in Scanning Transmission Electron Microscopy." Ultramicroscopy 108 (12):1653-58. https://doi.org/10.1016/j.ultramic.2008.07.001.

Lin, Yung Chang, Po Yuan Teng, Po Wen Chiu, and Kazu Suenaga. 2015. "Exploring the Single Atom Spin State by Electron Spectroscopy." Physical Review Letters 115 (20):1-5. https://doi.org/10.1103/PhysRevLett.115.206803.

Lovejoy, T. C., Q. M. Ramasse, M. Falke, A. Kaeppel, R. Terborg, R. Zan, N. Dellby, and O. L. Krivanek. 2012. "Single Atom Identification by Energy Dispersive X-Ray Spectroscopy." Applied Physics Letters 100 (15):0-4. https://doi.org/10.1063/1.3701598.

MacArthur, K E, L B Jones, and P.D. Nellist. 2014. "How Flat Is Your Detector? Non-Uniform Annular Detector Sensitivity in STEM Quantification." Journal of Physics: Conference Series 522 (1). IOP Publishing:12018. https://doi.org/10.1088/1742-6596/522/1/012018.

Malis, T, S C Cheng, and R F Egerton. 1988. "EELS Log-Ratio Technique for Specimen-Thickness Measurement in the TEM." Journal of Electron Microscopy Technique 8 (2):193-200. https://doi.org/10.1002/jemt.1060080206.

Martinez, G.T., Lewys Jones, A. De Backer, Armand Béché, J. Verbeeck, S. Van Aert, and P.D. Nellist. 2015. "Quantitative STEM Normalisation: The Importance of the Electron Flux." Ultramicroscopy 159 (P1):46-58. https://doi.org/10.1016/j.ultramic.2015.07.010.

Müller-Caspary, Knut, Oliver Oppermann, Tim Grieb, Florian F. Krause, A. Rosenauer, Marco Schowalter, Thorsten Mehrtens, et al. 2016. "Materials Characterisation by Angle-Resolved Scanning Transmission Electron Microscopy." Scientific Reports 6 (November). Nature Publishing Group:37146. https://doi.org/10.1038/srep37146.

Retsky, M. 1974. "Observed Single Atom Elastic Cross Sections in a Scanning Electron Microscope.” Optik. https://doi.org/10.2172/4239746. 
Rosenauer, A., Thorsten Mehrtens, Knut Müller, Katharina Gries, Marco Schowalter, Parlapalli Venkata Satyam, Stephanie Bley, et al. 2011. "Composition Mapping in InGaN by Scanning Transmission Electron Microscopy." Ultramicroscopy 111 (8):1316-27. https://doi.org/10.1016/j.ultramic.2011.04.009.

Singhal, A., J.C. Yang, and J.M. Gibson. 1997. "STEM-Based Mass Spectroscopy of Supported Re Clusters." Ultramicroscopy 67 (June):191-206. https://doi.org/10.1016/S0304-3991(96)00094-0.

Yamashita, Shunsuke, Shogo Koshiya, Kazuo Ishizuka, and Koji Kimoto. 2015. "Quantitative Annular Dark-Field Imaging of Single-Layer Graphene.” Microscopy 64 (2):143-50. https://doi.org/10.1093/jmicro/dfu115. 